\title{
IMPORTANCIA DEL PROFESIONAL DE LA INFORMACIÓN EN LA DIRECCIÓN DE COMUNICACIÓN DE LAS ORGANIZACIONES
}

\author{
Importance of the professional of the information in \\ the direction of communication in organizations
}

\section{María-Victoria Carrillo-Durán}

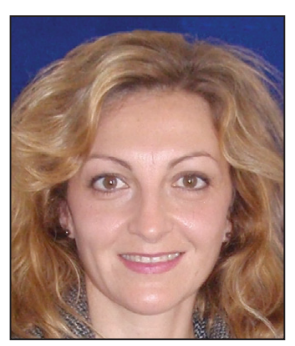

María-Victoria Carrillo-Durán es profesora titular de la Universidad de Extremadura. Imparte docencia en los grados de comunicación audiovisual y de información y documentación. Su investigación gira en torno a la comunicación en las organizaciones, habiendo publicado libros y artículos en esta materia y contando con dos sexenios de investigación. Algunas de sus publicaciones son: Los retos de la comunicación (Pearson, 2013) y Comunicación integral y responsabilidad social en las organizaciones (Pearson, 2009).

http://orcid.org/0000-0002-1256-8870

Universidad de Extremadura, Facultad de Ciencias de la Documentación y Comunicación Plaza Ibn Marwan, s/n. 06001 Badajoz, España vicduran@unex.es

\section{Resumen}

Se destaca la importancia del profesional de la información en la dirección de comunicación (dircom) de las organizaciones. Resulta fundamental la integración de un gestor de información en el equipo de trabajo del dircom, o bien que sea éste el que adquiera un perfil interdisciplinar con conocimientos de información. La dirección de comunicación no es ya sólo el departamento de prensa sino un gestor integral de la comunicación (tanto interna como externa), de los activos intangibles, y de las políticas de reputación, imagen y creación de valor a través de la marca. En este artículo se revisan las tareas demandadas por las empresas desde sus áreas de comunicación y su relación con las competencias de los profesionales de la información. Este trabajo es una aportación cross-fertilization que justifica el mapa de tareas que el profesional de la información puede realizar en materia de comunicación.

\section{Palabras clave}

Comunicación empresarial; Recursos intangibles; Gestión de la información; Director de comunicación; Profesional de la información.

\begin{abstract}
The importance of the information professional in the communication management department of organizations is discussed. The need to integrate an information specialist within the communication team is essential; either hired from outside the organization or trained from within. Communication management is no longer limited to a press department handling press releases, but is instead a comprehensive communication management effort dealing with internal and external relations; and policies about intangible assets like reputation, image, and value creation through brand. In this article we review the tasks that are demanded by companies of their communication departments and compare them with the skills of information professionals. This job requires a cross-fertilization effort and a task map is used to illustrate how information professionals can perform in the communication area.
\end{abstract}

\section{Keywords}

Organizational communication; Intangible assets; Information management; Communication manager; Professional of the information.

Carrillo-Durán, María-Victoria (2016). "Importancia del profesional de la información en la dirección de comunicación de las organizaciones". El profesional de la información, v. 25, n. 2, pp. 272-278.

http://dx.doi.org/10.3145/epi.2016.mar.14 


\section{Introducción}

Según Timoteo-Álvarez (2013) las etapas vividas en el desarrollo de la gestión de la comunicación actual van desde una fase meramente instrumental de la comunicación en los años ochenta, a una fase posterior en la que la comunicación se vuelve estratégica en los noventa, para preparar el terreno al modelo de gestión digital de referencia en la actualidad. El mismo autor (Timoteo-Álvarez, 2013, p. 2) evidencia así que la gestión de la comunicación vive hoy su tercera revolución.

Fruto de esta evolución, la dirección de comunicación hace tiempo que dejó de ser el departamento de prensa de las empresas, encargado de la comunicación externa hasta los años noventa del siglo pasado. El inicio de siglo trajo aparejado un cambio sustancial en las tareas y utilidad de ese órgano de gestión, que mudó hacia la gestión integral de la comunicación (tanto corporativa como interna), los activos intangibles, y las políticas de reputación, imagen y creación de valor a través de la marca (Victoria-Mas; Lacasa-Mas, 2015).

El director de comunicación (dircom) actual es un traductor de los objetivos y estrategias de gestión de la empresa en objetivos y políticas de comunicación al servicio de los activos intangibles (Carrillo-Durán; Nuño-Moral, 2010). En este panorama en el que ha habido que redefinir los roles y las tareas a desempeñar por la dirección de comunicación, la gestión de la información toma especial protagonismo (Campillo-Alhama, 2013).

Este artículo explora la incorporación del profesional de la gestión de la información en las áreas de dirección de la comunicación de las grandes empresas. Para ello nos apoyamos en diversos documentos que reflejan la realidad de la nueva gestión de la comunicación (como el informe de Burson-Masteller, 2013), en las aportaciones de otros autores, como Guallar (2012) y Guallar y Leiva-Aguilera (2013) y las competencias descritas en el documento Euro-referencial en información y documentación (ECIA, 2004) sobre los perfiles y competencias del profesional de la información. El enfoque transdisciplinar entre los dos campos de conocimiento (comunicación y documentación/información) constituye la principal aportación de este trabajo.

\section{El profesional de la comunicación estratégica y el profesional de la información}

La comunicación ya no es una labor menor en las organizaciones. La presencia de departamentos de comunicación en las grandes organizaciones no es una novedad, pero sí lo es el lugar que estas áreas están alcanzando en sus organigramas. Un ejemplo es el banco BBVA que tiene en su organigrama un Departamento de Comunicación directamente ligado al Área de Presidencia (BBVA, 2015). Otro ejemplo es el grupo Inditex que incluyó hace tiempo el área de comunicación corporativa dependiente de vicepresidencia (Salazar-Castillo, 2005), y eso que es bien sabido que las principales marcas de Inditex, como Zara, no realizan publicidad, lo que es un indicativo de que la gestión de la comunicación no está reducida a la gestión de la comunicación comercial, entendida básicamente como publicidad.
La gestión de la comunicación ha pasado de ser una tarea de marketing o incluso una labor periodística, a ser estratégica. Las organizaciones de hoy no demandan exclusivamente profesionales de la comunicación sino profesionales de la gestión de los activos intangibles por medio de la comunicación. El trabajo necesita responsables capaces de asumir el nuevo contexto y de desempeñar la labor fundamental de integrar la comunicación en la consecución de la estrategia general de la compañía (Carrillo-Durán, 2014).

El nuevo dircom es un profesional dedicado a la integración de las políticas de comunicación e información con el resto de políticas de la empresa

Se demanda hoy un perfil profesional que no coincide exactamente con el tradicional dircom como responsable de las acciones de comunicación aisladas, para cuya tarea era suficiente un perfil de formación en periodismo, comunicación audiovisual o publicidad y relaciones públicas. El nuevo dircom es un profesional especialmente dedicado a la integración de las políticas de comunicación y de información con el resto de políticas (Villafañe-Gallego, 1999), de modo que sus decisiones permitan contribuir al éxito de la empresa (Carrillo-Durán, 2014). Resulta fundamental la integración de un gestor de información en el equipo de trabajo del dircom o incluso que el perfil interdisciplinar del que hablábamos más arriba para el dircom asuma el conocimiento de esta tarea.
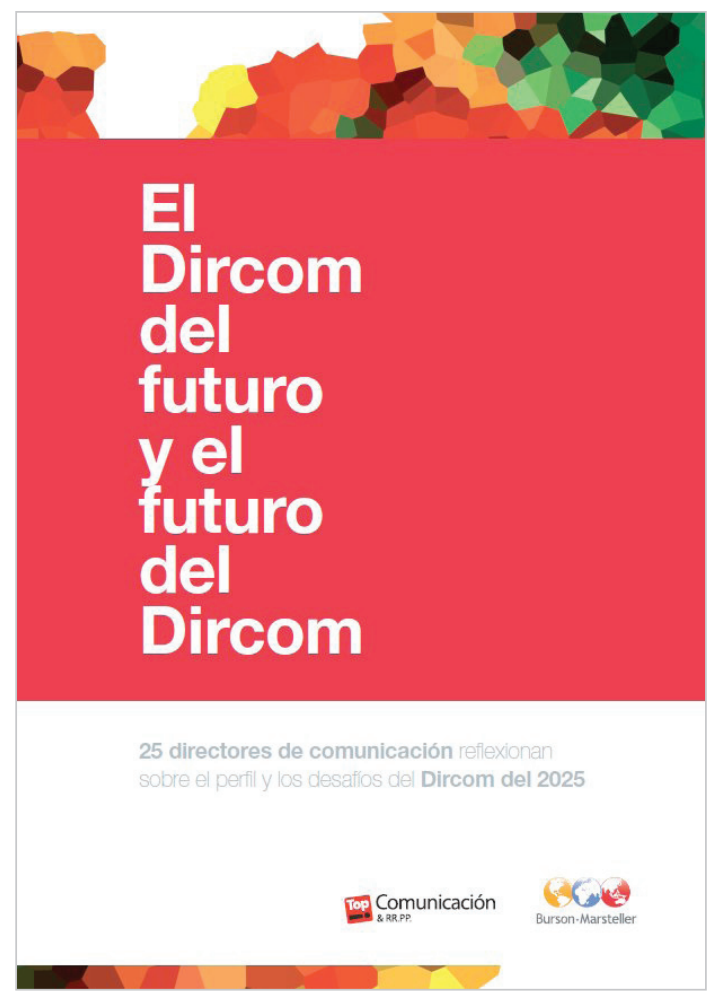

http://burson-marsteller.es/wp-content/uploads/2013/03/InformeFuturo-Dircom.pdf 
Tabla 1. Integración de competencias y perfiles profesionales desarrollados por niveles.

Fuente: Euro-referencial en información y documentación (ECIA, 2004, p. 84).

\begin{tabular}{|c|l|l|}
\hline Nivel & \multicolumn{1}{|c|}{ Cargo } & \multicolumn{1}{|c|}{ Funciones } \\
\hline 4 & Director de comunicación & $\begin{array}{l}\text { Alinear todas las políticas con la estrategia de comunicación. } \\
\text { Seguimiento y evaluación de la política de comunicación. } \\
\text { Desarrollar acciones para públicos internos y externos. }\end{array}$ \\
\hline 3 & Director de información & $\begin{array}{l}\text { Detectar necesidades informativas y hacer recomendaciones. } \\
\text { Negociar los medios necesarios. } \\
\text { Elaborar documentos (en todos los soportes) para promover sus actividades. }\end{array}$ \\
\hline 2 & Técnico de comunicación & $\begin{array}{l}\text { Personalizar la comunicación. } \\
\text { Colgar información en la intranet. } \\
\text { Reunir y preparar elementos para la comunicación. } \\
\text { Elegir medios técnicos. }\end{array}$ \\
\hline 1 & Técnico de información & \begin{tabular}{l} 
Identificar los productos de información y conocer el entorno de la comunicación. \\
\hline
\end{tabular}
\end{tabular}

Otro elemento destacable es la presencia en el panorama académico español de dobles grados en comunicación audiovisual e información y documentación (en la Universidad de Extremadura, Universidad de Barcelona, Universidad Carlos III de Madrid) y grado en periodismo e información y documentación de la Universidad de Murcia. El resultado es que los planes de estudios conducentes a estos títulos combinan enseñanzas útiles para los dircom del futuro, perfil que habría que combinar igualmente con la obtención de una completa formación empresarial.

\section{La comunicación ha pasado de ser una tarea de marketing o incluso de perio- dismo, a ser estratégica}

Si las razones académicas demuestran una útil integración de saberes, la práctica real constata igualmente la necesidad de un profesional de la información en la dirección de comunicación, dado que según Burson-Masteller (2013, p. 9) en el informe sobre el dircom del futuro previsto para el horizonte 2025:

"la gestión de la información, tanto externa como interna, es un capítulo de importancia crítica para la empresa, y es una función que no se puede realizar sin estar en puestos del más alto nivel".

Ante esta evidencia, y tal como expone la Sociedad Española de Documentación e Información Científica (Sedic, 2015):

"el desarrollo de la sociedad de la información ha potenciado la función que desempeñan dentro de las organizaciones las tareas de gestión de la información. Por ello se demandan cada vez más profesionales preparados y con experiencia en la organización del conocimiento, el tratamiento y la recuperación eficaz de información".

Para la Sedic, este colectivo está consolidado en España a nivel académico y profesional, ha demostrado su conocimiento de las tecnologías y sistemas de gestión de la información, y puede ser definido como un especialista en información encargado de satisfacer las necesidades informativas de la comunidad para la que trabaja, en este caso, la empresa y sus diferentes grupos de interés o stakeholders.

Parece que el profesional de la información contribuirá decisivamente a que el objetivo marcado para la dirección de comunicación del futuro se cumpla. El citado Euro-referencial en información y documentación (ECIA, 2004, p. 84) describe el conocimiento en comunicación institucional dentro de las competencias necesarias para el profesional de la información, definido como:

"concebir y realizar operaciones de comunicación con el fin de posicionar y promover sus actividades dentro y fuera de la empresa".

En la tabla 1 se resumen las competencias necesarias para alcanzar los cuatro niveles de desarrollo estimados y la implicación de cada nivel de formación del profesional de la información en las tareas de comunicación.

Como resumen de las correspondencias entre niveles competenciales y posibles perfiles profesionales, se considera que a medida que aumentamos de nivel, el trabajo del profesional de la información muda hacia el perfil de director de comunicación. Aunque las competencias descritas en todos los niveles (no sólo en el 4, más avanzado) se contienen en el perfil de un director de comunicación, éstas no son suficientes para ejercer este trabajo hoy en día. No obstante, si en la formación específica recibida por un profesional de la información se completan al menos estas competencias, aumentan sus posibilidades de desempeñar las tareas de dircom.

\section{Por qué necesitamos a un profesional de la información en la dirección de comunicación}

La necesaria implicación del gestor de información en las tareas de gestión de la comunicación ayuda a definir el nuevo perfil del dircom del futuro, que según Burson-Masteller (2013, p. 37):

"tendrá una función más estratégica, controlará los efectos de los nuevos canales, gestionará la reputación de la marca a nivel global, gestionará el gran volumen de información que se produce y repercute en la imagen de la empresa, solucionará las cada vez más frecuentes crisis online, responderá a las mayores exigencias de transparencia por parte de la sociedad y dialogará con grupos de interés cada vez más numerosos".

No parece posible este trabajo sin un equipo de profesionales de la información que ayuden en las tareas descritas, aportando sus conocimientos específicos a la solución de algunos de los problemas que se plantean a continuación y que debe enfrentar el área de comunicación (Burson-Masteller, 2013, p. 17). 


\subsection{Sobreabundancia informativa}

El trabajo del dircom ya no se puede realizar al margen del control de la información interna y externa, desplegada en torno a la organización. Para la directora de marketing y comunicación de Panda Security en Burson-Masteller (2013, p. 12-13):

"Cada vez hay más información disponible para el público, y esta información ya no es generada por la empresa, sino por el usuario, con lo que el papel del director de comunicación ya no se limita a lanzar los mensajes oficiales, sino sobre todo a gestionar, matizar, o minimizar la información que viene de los usuarios y otro tipo de fuentes no oficiosas".

Este volumen de información procede tanto de medios tradicionales como online (redes sociales, blogs, foros, etc.). La complejidad creada por las tecnologías de la información y la digitalización ha llegado para quedarse (Scolari, 2012) y nos llevará a idear maneras nuevas de gestionar la información y sistemas de análisis de big data.

Internet ha aportado complejidad a la tarea de control de la información. La necesidad de conocer técnicas de gestión específicas es razón suficiente para buscar un experto capaz de encontrar la información (dada la multiplicidad de canales y fuentes disponibles) y medir su relevancia (dada la multiplicidad de portavoces que tendrá la empresa en redes sociales y otros medios digitales).

La transparencia no es una característica opcional de la información sino una condición sine qua non

\subsection{Necesidad de transparencia informativa con cada grupo de interés}

En los últimos años ha aumentado la exigencia de transparencia en la tarea de gestión de la comunicación. Para la directora de Comunicación de Dow Chemical Ibérica para España y Portugal en Burson-Masteller (2013, p. 13):

"la sociedad es cada vez más transparente y las exigencias de los grupos de interés van más allá de la información corporativa básica".

Esta tarea exige la habilidad suficiente para implementar políticas de comunicación apoyadas en la veracidad de la información y en su adaptación a los diferentes grupos de interés.

No es suficiente contar el mensaje sino que hay que adaptarlo a los diferentes grupos y transmitirlo traspasando los filtros de la desconfianza y el descrédito generado por las empresas en los últimos tiempos. La transparencia no es una característica opcional de la información sino una condición sine qua non y es posible gestionarla al servicio de los recursos intangibles, tales como la reputación corporativa. Estando en juego ésta, es evidente que transparencia e información deben ir unidas.

Elaborar un plan estratégico de comunicación significa que ésta debe estar orientada a los grupos de interés, y evitar la confusión y la improvisación. La información no debe ser transparente sólo porque es la base de la política de comunicación, sino que debe adquirir las propiedades necesarias para construir valor en cada uno de los grupos de interés con los que se entabla la comunicación.

\subsection{Multiplicidad de fuentes de dudosa credibilidad}

Es otro reto para la gestión de la comunicación, en el que el profesional de la información tendrá un rol destacado. El dircom va a:

"tener que gestionar noticias cuyas fuentes pueden ser muy poco fiables y procedentes de cualquier lugar del mundo y que se replican, de forma inmediata, sin ningún tipo de contraste de su veracidad..." (Burson-Masteller, 2013, p. 17).

Esta situación tendrá a todo el equipo en permanente alerta ante las posibles crisis que se puedan desatar. La anticipación a estas situaciones va a depender en gran parte de la rapidez para detectar las fuentes calientes de información detonantes de situaciones controvertidas, y de la rapidez en la reacción para neutralizarlas. El profesional de la información tiene además que depurar la información de ruidos, apartando el mensaje nítido y la información útil de la cacofonía provocada por la ilimitada posibilidad de lanzar mensajes.

\subsection{Aumento de portavoces, líderes y grupos de in- terés}

La multiplicidad de grupos de interés será otro hecho de importancia trascendental. Si éstos se amplían -y ya no son sólo los clientes y los empleados-, se dificulta la gestión informativa. La presencia de múltiples grupos en el entorno de las organizaciones (accionistas, proveedores, medios de comunicación, clientes, empleados, órganos reguladores...) exige mayor control tanto de la cantidad de información precisa que necesita cada grupo, como de la información que puede generar cada uno de ellos.

La detección de estos grupos de interés y su análisis, desde el punto de vista de cómo afectan a los intereses de la empresa (cómo afectan a la opinión pública, a la imagen de la empresa, a sus intereses económicos, a la imagen comercial de los productos...) repercutirá en las decisiones de comunicación y en la cantidad precisa de información que hay que generar para cada grupo (Villafañe-Gallego, 1999), lo que requerirá la preparación de información mucho más personalizada.

Por otra parte, cada grupo de interés delimitado se convierte en una fuente de información de interés para la organización, que puede afectarla en positivo o en negativo. De ahí la necesidad de controlar su potencial informativo como fuentes y de definir, tal y como ya se venía haciendo, el trabajo de comunicación a desarrollar con cada uno de ellos.

Algunos de los grupos de interés más frecuentes en las organizaciones pueden variar su rol. Los medios de comunicación y los periodistas mantendrán su importancia, aunque verán reducido su margen de acción por la presencia de nuevos "líderes de opinión" (blogueros, tuiteros de referen- 
Tabla 2. Relación entre las áreas de conocimiento del dircom y algunos perfiles profesionales de información y documentación

\begin{tabular}{|c|c|c|}
\hline $\begin{array}{l}\text { Perfil del nuevo dircom } \\
\text { Burson-Masteller (2013) }\end{array}$ & $\begin{array}{l}\text { Perfiles entre la comunica- } \\
\text { ción y la documentación }\end{array}$ & Aportación al perfil del dircom \\
\hline $\begin{array}{l}\text { Gestor de estrategia empresarial. } \\
\text { El nuevo dircom que trabaja en la empresa al más } \\
\text { alto nivel debe entender los principios de la gestión } \\
\text { empresarial y las bases de las políticas de la organiza- } \\
\text { ción, controlando las fuentes y los recursos internos } \\
\text { y externos. }\end{array}$ & $\begin{array}{l}\text { Responsable de servicios de } \\
\text { búsqueda y organización de } \\
\text { la información en diferentes } \\
\text { fuentes o recursos internos y } \\
\text { externos (Sedic, 2015). }\end{array}$ & $\begin{array}{l}\text { Conocimiento necesario para la gestión de la informa- } \\
\text { ción empresarial en la toma de decisiones de comu- } \\
\text { nicación como parte fundamental de la gestión de la } \\
\text { empresa en su conjunto. }\end{array}$ \\
\hline $\begin{array}{l}\text { Gestor de intangibles. } \\
\text { El trabajo del dircom debe asumir la gestión de intan- } \\
\text { gibles, lo cual obliga a contar con otros profesionales } \\
\text { que ayuden en áreas específicas de la gestión de } \\
\text { estos recursos, tales como la gestión del conocimien- } \\
\text { to, la reputación online o la responsabilidad social } \\
\text { corporativa (Aceituno-Aceituno et al., 2013). }\end{array}$ & $\begin{array}{l}\text { Gestor de la reputación online } \\
\text { (Guallar, 2012). } \\
\\
\text { Responsable de sistemas de } \\
\text { gestión del conocimiento } \\
\text { (Pinto-Prieto; Becerra-Ardila; } \\
\text { Gómez-Flórez, 2013) }\end{array}$ & $\begin{array}{l}\text { Conocimiento de la gestión de la información digital } \\
\text { que ayudará a completar las necesidades de ubicación } \\
\text { y seguimiento de las informaciones digitales sobre la } \\
\text { empresa. } \\
\text { Saber sobre los sistemas de gestión del conocimiento } \\
\text { (SGC) propiciando los "procesos de generación, codifi- } \\
\text { cación y transferencia del conocimiento en las organiza- } \\
\text { ciones" (Pinto-Prieto; Becerra-Ardila; Gómez-Flórez, } \\
2013, \text { p. 1). }\end{array}$ \\
\hline $\begin{array}{l}\text { Experto en herramientas } 2.0 \text {. } \\
\text { El dircom debe contar con un amplio conocimiento } \\
\text { de la tecnología y de la comunicación } 2.0 \text {. }\end{array}$ & $\begin{array}{l}\text { Community manager (Marqui- } \\
\text { na-Arenas, 2012). }\end{array}$ & $\begin{array}{l}\text { Detectar las principales comunidades y sus líderes de } \\
\text { opinión, escuchar y seguir sus conversaciones. Iniciar } \\
\text { conversaciones y generar contenidos informativos } \\
\text { adaptados, sabiendo involucrar a los participantes en la } \\
\text { dirección correcta (Marquina-Arenas, 2012). } \\
\text { Este profesional deberá entender y manejar las técnicas } \\
\text { de SEO, SEM y analítica web que faciliten un mejor posi- } \\
\text { cionamiento en los buscadores. }\end{array}$ \\
\hline $\begin{array}{l}\text { Gestor de contenidos. } \\
\text { A través del marketing de contenidos y el branded } \\
\text { journalism (periodismo de marca) los dircom de hoy } \\
\text { deben convertir las marcas-empresas en cabeceras de } \\
\text { prensa digital, con portales informativos internacio- } \\
\text { nales y con contenidos de calidad. }\end{array}$ & $\begin{array}{l}\text { Documentalista-periodista y } \\
\text { content curator (Guallar, 2012). }\end{array}$ & $\begin{array}{l}\text { Técnicas para generar contenidos relevantes y dominar } \\
\text { la creación de contenidos online que respalden mejor } \\
\text { los mensajes de marca, aumentando así su protagonis- } \\
\text { mo profesional en los departamentos de comunicación } \\
\text { (García-Gutiérrez; Martínez-Ávila, 2014). } \\
\text { La aportación del content curator (Guallar; Leiva-Agui- } \\
\text { lera, 2013) es mantener la relevancia de la información } \\
\text { y hacer una selección adaptada para la empresa, con } \\
\text { el contenido y recursos más adecuados a sus intereses, } \\
\text { no siendo posible este trabajo para alguien que no sea } \\
\text { documentalista. }\end{array}$ \\
\hline $\begin{array}{l}\text { Experto en técnicas de medición de la información } \\
\text { sobre todo en las webs. } \\
\text { La actitud de escucha activa trae aparejado el análisis } \\
\text { e interpretación de las informaciones a través de } \\
\text { diferentes métricas. }\end{array}$ & $\begin{array}{l}\text { Experto en informetría (web- } \\
\text { metría). }\end{array}$ & $\begin{array}{l}\text { Métrica de la información: bibliometría, webmetría, } \\
\text { cibermetría, altmetría, indicadores, índices de impacto, } \\
\text { mercado de la información, estadísticas de la informa- } \\
\text { ción, evaluación cuantitativa, volumen de datos, valor } \\
\text { de la información, análisis de calidad y relevancia, uso, } \\
\text { pertinencia y exhaustividad de los servicios y sistemas de } \\
\text { información, y visualización de datos. }\end{array}$ \\
\hline $\begin{array}{l}\text { Gestor de información e inteligencia competitiva. } \\
\text { Este conocimiento permitirá el análisis de la informa- } \\
\text { ción para extraer conclusiones más estratégicas que } \\
\text { las del análisis de los medios con las herramientas } \\
\text { tradicionales, basadas en la cobertura y la frecuencia } \\
\text { del mensaje. }\end{array}$ & $\begin{array}{l}\text { Fact checker o comprobador de } \\
\text { datos (Guallar, 2012) }\end{array}$ & $\begin{array}{l}\text { El fact checker o comprobador de datos (Guallar, 2012) } \\
\text { verifica la información y su capacidad de influencia, tarea } \\
\text { que está subyacente en el perfil del periodista y que se } \\
\text { ve completada por este profesional documentalista. }\end{array}$ \\
\hline $\begin{array}{l}\text { Experto en neurociencia y psicología de masas. } \\
\text { Resulta necesario entender muy bien las motivacio- } \\
\text { nes, inquietudes y frustraciones de los grupos de inte- } \\
\text { rés para llegar a ellos con los mensajes corporativos. }\end{array}$ & $\begin{array}{l}\text { Responsable de proyectos de } \\
\text { análisis y evaluación de pro- } \\
\text { ducción, demanda o consumo } \\
\text { de información, mediante } \\
\text { técnicas cuantitativas y cuali- } \\
\text { tativas (Sedic, 2015). }\end{array}$ & $\begin{array}{l}\text { Conocimientos sobre el consumo de información de } \\
\text { cada grupo de interés, para ayudar a determinar sus } \\
\text { hábitos y formas de comportamiento en su relación con } \\
\text { los medios. }\end{array}$ \\
\hline $\begin{array}{l}\text { Gestor de crisis, en especial en entornos online. } \\
\text { La gestión de crisis es una tarea de la que conoce } \\
\text { bastante el dircom. Es fundamental la especialización } \\
\text { en la forma de gestionar la repercusión de las crisis en } \\
\text { el entorno online. }\end{array}$ & $\begin{array}{l}\text { Community manager y gestor } \\
\text { de la reputación online (Gua- } \\
\text { llar, 2012) }\end{array}$ & $\begin{array}{l}\text { Detectar fuentes de información negativa, establecer su } \\
\text { alcance y neutralizarlas. }\end{array}$ \\
\hline $\begin{array}{l}\text { Experto en la evaluación de la imagen y otros } \\
\text { recursos intangibles a través de las auditorías de } \\
\text { información y de comunicación. (Carrillo-Durán; } \\
\text { Nuño-Moral, 2010). } \\
\text { Las auditorías permiten elaborar diagnósticos y } \\
\text { valorar los planes estratégicos de comunicación que } \\
\text { haya que desarrollar para corregir las desviaciones en } \\
\text { torno a la imagen y la reputación corporativas. }\end{array}$ & $\begin{array}{l}\text { Responsable de proyectos de } \\
\text { creación y mantenimiento de } \\
\text { sistemas de información y de } \\
\text { bases de datos documentales } \\
\text { (Sedic, 2015). }\end{array}$ & $\begin{array}{l}\text { Facilitar el acceso a la información construida sobre } \\
\text { bases de datos empíricas, rigurosas y de calidad, para } \\
\text { gestionar la toma de decisiones sobre la reputación y } \\
\text { la marca, velando por la buena imagen corporativa y la } \\
\text { creación de valor. }\end{array}$ \\
\hline
\end{tabular}


cia, etc.). Este panorama de segmentación y atomización, con grupos de interés casi unipersonales, decantará a las organizaciones por un profesional de la información capaz de identificar, seguir y valorar la calidad informativa de cada grupo como fuente.

\section{Perfil del nuevo dircom y de los profesionales de la información y la documentación}

Parece incuestionable que el dircom debe tener un perfil mucho más abierto y completo que el que tenía hasta hace pocos años. Su tarea se ha complejizado y requiere de unos conocimientos interdisciplinares que pueden ser asumidos por él mismo o por un profesional de la información y la documentación en su equipo de trabajo.

Según los perfiles que se dibujan a medio camino entre la comunicación y la documentación [Guallar (2012), Marquina-Arenas (2012), Sedic (2015)], y las áreas de conocimiento necesarias para completar el mapa competencial del dircom (Burson-Masteller, 2013), es posible establecer las relaciones entre lo que pueden aportar esos nuevos perfiles de la documentación y la información al complemento de formación necesaria para desarrollar la tarea de dircom (tabla 2).

Existe pues una relación entre los conocimientos necesarios para el dircom del futuro y las aportaciones de los perfiles profesionales que se configuran a medio camino entre el profesional de la documentación/información y la comunicación.

Perfiles como los de community manager y gestor de la reputación online deberán ser, además de buenos profesionales de la comunicación, buenos profesionales de la información

\section{Conclusiones y líneas de atención futuras}

De este recorrido se pueden extraer algunas ideas que perfilan nuevos horizontes para los profesionales de la información en las direcciones de comunicación de las grandes empresas preocupadas por las nuevas áreas de gestión de recursos intangibles y comunicación.

En primer lugar, aumenta la demanda de profesionales de la información por los conocimientos que pueden aportar de acuerdo con los nuevos servicios requeridos por las organizaciones y a las nuevas competencias que debe adquirir el dircom (director de comunicación).

Se incrementa la demanda de servicios de consultoría estratégica de la comunicación. El dircom del pasado era un experto al servicio de las acciones de comunicación corporativa e interna de las empresas. Hoy en día la función de comunicación se ha convertido en estratégica y se ha elevado en los organigramas, siendo el paraguas que respalda las acciones de otras áreas de gestión de las firmas, de modo que las decisiones de los departamentos tradicionales tendrán una repercusión en la imagen de la empresa y deben ser controladas en conjunto a nivel comunicativo.
El nuevo dircom necesitará ser asesorado en viejas y nuevas áreas estratégicas de la gestión empresarial, en las que el control de la información que se genera en ellas es obligado. De acuerdo con este nuevo entorno, las agencias o consultorías de comunicación y los departamentos de comunicación de las empresas deben contar con un grupo cada vez más interdisciplinar de ayuda a la gestión estratégica de la comunicación. En esta línea Burson-Masteller (2013, p. 24) señala que "muchas agencias crearán departamentos de I+D+i para ponerlos al servicio de sus clientes". Sean departamentos de I+D o interdisciplinares, perfiles profesionales como los responsables de sistemas de información y de bases de datos documentales o responsables de servicios de búsqueda y organización de la información en diferentes fuentes o recursos internos y externos (Sedic, 2015) serán bien acogidos para este servicio.

Perfiles como los de periodista-documentalista o content curator serán muy apreciados en las nuevas áreas de gestión de la comunicación

Aumenta el interés por las nuevas áreas de gestión de intangibles, tales como la reputación, que no pueden vivir al margen del entorno 2.0. Dado que las empresas no tienen una reputación online y otra offline, el control de la reputación online ya no es una opción, sino una obligación fundamental para la toma de decisiones que afectan a la gestión de la reputación corporativa en su conjunto. Las empresas y las agencias de comunicación se especializarán en el control de la información digital en las principales plataformas de social media, por lo que perfiles como los de community manager y gestor de la reputación online (Guallar, 2012) deberán ser, además de buenos profesionales de la comunicación, buenos profesionales de la información.

Se eleva la demanda de servicios de comunicación de áreas especializadas. Ya no es posible asesorar en materia de comunicación aplicando una serie de principios básicos y homogéneos. La comunicación se ha vuelto financiera, sanitaria, política..., por lo que el dircom debe también diferenciar su tarea en la empresa de la que se realiza en otras organizaciones. La calidad de los servicios específicos ofrecidos trae aparejada la personalización de la información, el análisis de datos específicos y de saberes y ciencias diversas, la evaluación de tendencias informativas y la medición de la información. En este caso, contar en los equipos con responsables de proyectos de análisis y evaluación de producción, demanda o consumo de información, mediante técnicas cuantitativas y cualitativas (Sedic, 2015), fact checkers o comprobadores de datos (Guallar, 2012) o expertos en informetría, contribuirá a la calidad de los servicios específicos demandados.

Se incrementa la demanda de servicios de creación de contenidos especializados para diferentes grupos de interés. Las nuevas organizaciones preocupadas por sus activos intangibles deben ser expertas en la generación de nuevos documentos y en la gestión de contenidos, que se convertirán en instrumentos de comunicación y en oportunidades para 
ofrecer el mensaje corporativo (Eíto-Brun, 2013; Fernández-Sande; Rodríguez-Barba; Rodríguez-Pallares, 2013). La búsqueda de la información adaptada y precisa para construir los mensajes corporativos y la redacción de programas, normas, manuales e informes, con fines comunicativos y no como meros documentos internos, requiere la habilidad de un comunicador y de un documentalista o profesional especializado en la gestión de la información. Perfiles tales como los de periodista-documentalista o content curator (Guallar, 2012) serán muy apreciados en las nuevas áreas de gestión de la comunicación y gestión de intangibles de las grandes empresas.

\section{Bibliografía}

Aceituno-Aceituno, Pedro; Cea-Moure, Ramiro; CasadoSánchez, José-Luis; Ruiz-de-Azcárate-Varela, Carmen (2013). "La comunicación como factor clave en la implantación de la responsabilidad social corporativa: el caso de Crédit Agricole España". El profesional de la información, v. 22, n. 4, pp. 326-332.

http://dx.doi.org/10.3145/epi.2013.jul.08

BBVA (2015). Organigrama.

http://accionistaseinversores.bbva.com/TLBB/tlbb/bbvair/ esp/group/chart/index.jsp

Burson-Masteller (2013). El dircom del futuro y el futuro de la comunicación. 25 directores de comunicación reflexionan sobre el perfil y los desafios del dircom del 2025. BursonMarsteller; Top Comunicación \& RR.PP.

http://burson-marsteller.es/wp-content/uploads/2013/03/ Informe-Futuro-Dircom.pdf

Campillo-Alhama, Concepción (2013). “Gestión de la información y su evaluación en los gabinetes de comunicación municipal". El profesional de la información, v. 22, n. 6, pp. 515-521.

http://dx.doi.org/10.3145/epi.2013.nov.03

Carrillo-Durán, María-Victoria (2014). “La comunicación estratégica y sus profesionales". Revista mediterránea de comunicación, v. 5, n. 2, pp. 33-46.

http://dx.doi.org/10.14198/MEDCOM2014.5.2.04

Carrillo-Durán, María-Victoria; Nuño-Moral, María-Victoria (2010). "La documentación en la evaluación y gestión de la imagen corporativa". El profesional de la información, v. 19, n. 2 , pp. $123-132$.

http://dx.doi.org/10.3145/epi.2010.mar.02

ECIA, European Council of Information Associations (2004). Euroreferencial en información y documentación. Competencias y aptitudes de los profesionales europeos de información y documentación. Volumen 1, Madrid: Sedic. ISBN: 8460936341

http://www.certidoc.net/es1/euref1-espanol.pdf

Eíto-Brun, Ricardo (2013). "Madurez de la gestión de contenidos, ¿sinónimo de desgaste o de oportunidades?". El profesional de la información, v. 22, n. 5, pp. 377-380. http://dx.doi.org/10.3145/epi.2013.sep.01
Fernández-Sande, Manuel; Rodríguez-Barba, Dolores; Rodríguez-Pallares, Miriam (2013). "La gestión de contenidos como actividad estratégica en empresas de radiodifusión. Estudio de casos en la radio comercial española". El profesional de la información, v. 22, n. 5, pp. 392-398.

http://dx.doi.org/10.3145/epi.2013.sep.03

García-Gutiérrez, Antonio; Martínez-Ávila, Daniel (2014). "Formación crítica de documentalistas en medios de comunicación". El profesional de la información, v. 23, n. 5, pp. 493-500.

http://dx.doi.org/10.3145/epi.2014.sep.06

Guallar, Javier (2012). "Perfiles profesionales en información y documentación. Panorámica global y nuevos roles entre la información y la comunicación". Seminario El mercado de trabajo en entornos digitales en información y documentación, Universidad de Zaragoza, 24 octubre.

http://eprints.rclis.org/19930

Guallar, Javier; Leiva-Aguilera, Javier (2013). El content curator. Guía básica para el nuevo profesional de internet. Barcelona: Editorial UOC, colección El profesional de la información, n. 24 . ISBN: 9788490640180

Marquina-Arenas, Julián (2012). Plan social media y community manager. Barcelona: Editorial UOC, colección El profesional de la información, n. 12. ISBN: 9788490292396

Pinto-Prieto, Laura-Patricia; Becerra-Ardila, Luis-Eduardo; Gómez-Flórez, Luis-Carlos (2012). "Carencias en los sistemas de gestión del conocimiento: una revisión bibliográfica". El profesional de la información, v. 21, n. 3, pp. 268-276. http://dx.doi.org/10.3145/epi.2012.may.07

Salazar-Castillo, José-Manuel (2005). "La estructura organizativa en la empresa actual. El caso Inditex". Boletín económico de ICE, n. 2850, 1 al 14 de agosto, pp.7-20 http://www.revistasice.com/CachePDF/BICE_2850_0718_C7E60DDC772602C905F583207547C14E.pdf

Scolari, Carlos A. (2012). "Comunicación digital. Recuerdos del futuro". El profesional de la información, v. 21, n. 4, pp. 337-340.

http://dx.doi.org/10.3145/epi.2012.jul.01

Sedic (2015). Nuestra profesión. Madrid: Área de Formación de la Sociedad Española de Documentación e Información Científica, Fosedic.

http://blog.sedic.es/category/articulos/nuestra-profesion

Timoteo-Álvarez, Jesús (2013). El manejo de la comunicación organizacional. Madrid: Díaz de Santos. ISBN: 978 8499694474

Victoria-Mas, María; Lacasa-Mas, Iván (2015). “Gestión del valor de marca en las empresas de prensa. El caso de La vanguardia". El profesional de la información, v. 24, n. 4, pp. 405-412. http://dx.doi.org/10.3145/epi.2015.jul.07

Villafañe-Gallego, Justo (1999). La gestión profesional de la imagen corporativa. Madrid: Pirámide. ISBN: 978 8436813845 